University of Wollongong

Research Online

Faculty of Engineering and Information

Faculty of Engineering and Information

Sciences - Papers: Part A

Sciences

$1-1-2013$

\title{
Microstructure and texture evolution in a twinning-induced-plasticity steel during uniaxial tension
}

Ahmed A. Saleh

University of Wollongong, asaleh@uow.edu.au

Elena V. Pereloma

University of Wollongong, elenap@uow.edu.au

Azdiar A. Gazder

University of Wollongong, azdiar@uow.edu.au

Follow this and additional works at: https://ro.uow.edu.au/eispapers

Part of the Engineering Commons, and the Science and Technology Studies Commons

Research Online is the open access institutional repository for the University of Wollongong. For further information contact the UOW Library: research-pubs@uow.edu.au 


\title{
Microstructure and texture evolution in a twinning-induced-plasticity steel during uniaxial tension
}

\begin{abstract}
A combination of electron back-scattering diffraction and X-ray diffraction was used to track the evolution of the microstructure and texture of a fully recrystallized Fe-24 Mn-3 Al-2 Si-1 Ni-0.06 C twinning-inducedplasticity steel during interrupted uniaxial tensile testing. Texture measurements returned the characteristic double fibre texture for face-centred cubic materials, with a relatively stronger $\langle 111\rangle$ and a weaker $\left\langle\begin{array}{lll}1 & 0 & 0\end{array}\right\rangle$ partial fibre parallel to the tensile axis. The interaction with the stable $\langle 111\rangle$ oriented grains results in preferential plastic flow in the unstable $\langle 110\rangle$ oriented grains. Consequently, the grains oriented along the $\langle 110\rangle$ and $\langle 100\rangle$ fibres record the highest and lowest values of intragranular local misorientation, respectively. The viscoplastic self-consistent model was used to simulate the macroscopic stress-strain response as well as track the evolution of bulk crystallographic texture by detailing the contributions of perfect and/or partial slip, twinning and latent hardening. The simulations revealed the dominant role of perfect slip and the limited volume effect of twinning on the texture development. The effects of initial orientation and grain interaction on the overall orientation stability during uniaxial tension showed that while the $\left\langle\begin{array}{lll}1 & 0 & 0\end{array}\right\rangle$ fibre remains stable and does not affect the unstable orientations along the $\langle 110\rangle$ fibre, the orientations along the stable $\left\langle\begin{array}{lll}1 & 1 & 1\end{array}\right\rangle$ fibre strongly affect the unstable $\langle 110\rangle$ orientations.
\end{abstract}

\section{Keywords}

induced, steel, during, uniaxial, twinning, plasticity, evolution, tension, microstructure, texture

Disciplines

Engineering | Science and Technology Studies

\section{Publication Details}

Saleh, A. A., Pereloma, E. V. \& Gazder, A. A. (2013). Microstructure and texture evolution in a twinninginduced-plasticity steel during uniaxial tension. Acta Materialia, 61 (7), 2671-2691.

This journal article is available at Research Online: https://ro.uow.edu.au/eispapers/1012 


\title{
MICROSTRUCTURE AND TEXTURE EVOLUTION IN A TWINNING INDUCED PLASTICITY STEEL DURING UNIAXIAL TENSION
}

\author{
Ahmed A. Saleh ${ }^{1 *}$, Elena V. Pereloma ${ }^{1,2}$, Azdiar A. Gazder ${ }^{1,2}$ \\ ${ }^{1}$ School of Mechanical, Materials and Mechatronic Engineering, University of Wollongong, New South \\ Wales 2522, Australia
}

2 Electron Microscopy Centre, University of Wollongong, New South Wales 2522, Australia

\begin{abstract}
A combination of Electron Back-Scattering Diffraction and X-ray diffraction was used to track the evolution of the microstructure and texture of a fully recrystallised Fe-24Mn-3Al-2Si-1Ni-0.06C TWinning Induced Plasticity (TWIP) steel during interrupted uniaxial tensile testing. Texture measurements returned the characteristic double fibre texture for fcc materials; with a relatively stronger $\langle 111\rangle$ and a weaker $\langle 100\rangle$ partial fibre parallel to the tensile axis. The interaction with the stable $\langle 111\rangle$ oriented grains results in preferential plastic flow in the unstable $\langle 110\rangle$ oriented grains. Consequently, the grains oriented along the $\langle 110\rangle$ and $\langle 100\rangle$ fibres record the highest and lowest values of intragranular local misorientation, respectively. The Visco-Plastic Self-Consistent (VPSC) model was used to simulate the macroscopic stress-strain response as well as track the evolution of bulk crystallographic texture by detailing the contributions of perfect and/or partial slip, twinning and latent hardening. The simulations revealed the dominant role of perfect slip and the limited volume effect of twinning on the texture development. The effects of initial orientation and grain interaction on the overall orientation stability during uniaxial tension showed that while the $\langle 100\rangle$ fibre remains stable and does not affect the unstable orientations along the $\langle 110\rangle$ fibre, the orientations along the stable $\langle 111\rangle$ fibre strongly affect the unstable $\langle 110\rangle$ orientations.
\end{abstract}

Keywords: TWIP, Electron back-scattering diffraction (EBSD), X-ray Diffraction, VPSC, texture, tension.

* Corresponding author: Ahmed A. Saleh

Phone: +61 $42215493 \quad$ Fax: +61 $42213662 \quad$ e-mail: asaleh@uow.edu.au 


\section{Introduction}

TWinning Induced Plasticity (TWIP) steels containing 25-35 wt.\% Mn with small additions of Al and Si are stable face centred cubic (fcc) austenitic steels with low stacking fault energy $\left(15-40 \mathrm{~mJ} / \mathrm{m}^{2}\right.$ ) [1]. Upon the imposition of external strain, the low stacking fault energy values promote deformation accommodation via the dissociation of perfect dislocations into partials and twinning.

It is well established that the uniaxial tensile deformation of fcc materials results in the development of a $\langle 111\rangle$ and $\langle 100\rangle$ double fibre texture parallel to the loading axis. The relative volume fraction of the two fibres generally depends on the value of stacking fault energy of the material. Following English and Chin [2], three generalised ranges have been identified such that: (i) high stacking fault energy materials exhibit a dominant $\langle 111\rangle$ fibre which is usually ascribed to the ease of cross-slip [2]. (ii) For intermediate to low stacking fault energy materials, the volume fraction of the $\langle 100\rangle$ fibre increases gradually with lower stacking fault energy values. The increase in the fraction of the $\langle 100\rangle$ fibre is thought to be associated with a volume effect of deformation twinning wherein the $\langle 111\rangle$ orientations reorient by twinning to the intermediate $\langle 115\rangle$ orientation. The $\langle 115\rangle$ orientations subsequently rotate by slip towards the $\langle 100\rangle$ orientation [3]. In disagreement with point (ii) of English and Chin, Stout et al. [4] argued that the stacking fault energy has a minor effect on the relative volume fraction of the $\langle 111\rangle$ and $\langle 100\rangle$ fibres. They suggested that the increase in the fraction of the $\langle 100\rangle$ fibre observed in [2] is probably due to the presence of a strong initial texture. (iii) With a further decrease in the value of the stacking fault energy and in behaviour that is reminiscent of high stacking fault energy materials, the volume fraction of the $\langle 100\rangle$ fibre decreases while the volume fraction of the $\langle 111\rangle$ fibre again increases. In this regard, a possible explanation for the strengthening of the $\langle 111\rangle$ fibre is the variation in the hardening on the active and latent slip systems with the change in stacking fault energy values. Here a lowering of the stacking fault energy tends to increase the latent hardening on the non-coplanar systems; which in turn promotes coplanar slip and results in an overall weakening of the $\langle 100\rangle$ fibre [2]. Another proposed mechanism for the weakening of the $\langle 100\rangle$ fibre involves the slip of individual partial dislocations which is associated with heavy deformation faulting in low stacking fault energy materials [5]. Earlier experimental investigations detailing the uniaxial tensile loading of TWIP steels have returned the typical fcc double fibre texture along with a relatively stronger $\langle 111\rangle$ fibre [6-9].

Whereas physical models have been developed to correlate the strain hardening behaviour with the TWIP effect [10-12], limited modelling efforts have been directed towards correlating the texture evolution with the microscopic deformation mechanisms. Very recently, Barbier et al. [13] used the Taylor and the 'translated-field' models to simulate the mechanical response, twinning activity and texture evolution during the tensile and shear loading of a Fe-22Mn-0.6C TWIP steel. Compared to the 
Taylor model, the 'translated-field' model (which is based on the self-consistent scheme) provided better agreement with the experimental data. Since this study did not account for the effect of twinning on the texture evolution, the good agreement with the experimental texture was ascribed to the dominant effect of slip on the evolution of the bulk texture [13].

While accounting for both slip and deformation twinning, Dancette et al. [14] applied a 'full-field' crystal plasticity - finite element method (CP-FEM) and a 'mean-field' multi-site model (which is an elastic-viscoplastic extension of the ALAMEL model [15]) to simulate the texture and hardening behaviour of a Fe-20Mn-1.2C TWIP steel during tensile loading and cold rolling. Once again, both these models returned improved texture predictions compared to the Taylor model. However, the multi-site model was found to be satisfactory only at moderate strains (30\% in tension) while the CP-FEM provided better texture simulations at higher strains (cold rolling to $54 \%$ thickness reduction). This was attributed to the failure of the short-range grain interactions (where each grain only interacts with its direct neighbours) in the multi-site model to capture the more complex deformation patterns expected at higher strains.

An alternate 'mean-field' approach is the widely used Visco-Plastic Self-Consistent (VPSC) plasticity model, which is based on the long-range interactions of individual grains with the polycrystalline aggregate [16-18]. Prakash et al. [19] employed the VPSC model to investigate the effect of deformation twinning on the texture evolution of a TWIP steel after tensile loading by applying the Predominant Twin Reorientation [20] and Kalidindi [21] twinning schemes. In that study, limited correspondence between the experimental and simulated stress-strain and texture data was obtained. Thereafter, Yan et al. [22] applied the VPSC model to predict the texture evolution after the tensile loading of Fe-25Mn-3Al-3Si TWIP steel such that the intensity of the experimental $\langle 111\rangle$ fibre was atypically under predicted and ascribed to the over prediction of twinning activity.

When both slip and twinning deformation modes were included in the VPSC model for the tensile loading of a Fe-18Mn-1.5Al-0.6C TWIP steel, De Cooman et al. [23] obtained over and under predictions of the $\langle 100\rangle$ and $\langle 111\rangle$ fibres, respectively. Once again, reasonable correlations between the experimental and VPSC-simulated texture was achieved only when the deformation was assumed to be accommodated solely via slip.

To this end, the present study tracks the microstructure and texture evolution during interrupted tensile loading of a fully recrystallised TWIP steel via a combination of X-ray Diffraction (XRD) and selected area Electron Back-Scattering Diffraction (EBSD). The influence of grain orientation on the development of intragranular local misorientation is discussed.

In contrast to the rather limited correspondence between the experimental and simulated results achieved in earlier self-consistent studies $[19,22,23]$, the present modelling work is the first to more successfully employ the VPSC model to simulate the macroscopic stress-strain response and the 
texture evolution during the uniaxial tensile loading of a TWIP steel. It is also the first study to assess the contributions of perfect and/or partial slip, twinning and latent hardening to the texture evolution in TWIP steel. Furthermore, the effects of initial orientation and grain interaction on the overall orientation stability during uniaxial tension was analysed with the aid of the VPSC model.

\section{Experimental and analytical procedure}

TWIP steel with the nominal composition $24 \mathrm{Mn}-3 \mathrm{Al}-2 \mathrm{Si}-1 \mathrm{Ni}-0.06 \mathrm{C}$ wt.\% was slab cast, hot rolled at $1100{ }^{\circ} \mathrm{C}$ to $52 \%$ thickness reduction and cold rolled to $42 \%$ thickness reduction. A flat dog-boned shaped tensile sample of $25 \mathrm{~mm}$ gage length, $5 \mathrm{~mm}$ width and $1 \mathrm{~mm}$ thickness was wire-cut from the centre of the cold rolled strip. The gage length and width of the tensile sample was parallel to the rolling (RD) and transverse (TD) directions of the cold rolled strip, respectively.

Following this, the tensile sample was isochronally annealed at $850{ }^{\circ} \mathrm{C}$ to its fully recrystallised state [24]. The heat treatment included $240 \mathrm{~s}$ of heating to stable temperature followed by $300 \mathrm{~s}$ of soaking time and immediate water quenching. Thereafter, the tensile sample was mechanically polished up to colloidal silica stage.

Uniaxial tensile testing was undertaken on an in-house modified Kammrath \& Weiss GmbH tensile stage operating in speed control mode at $5 \mu \mathrm{m} . \mathrm{s}^{-1}$. The test was interrupted at pre-determined loads that correspond to true strains of $0.14 \%, 2.3 \%, 7.2 \%, 20.9 \%, 32.3 \%$ and $48 \%$. The tensile strain of $48 \%$ also corresponds to the ultimate tensile strength of the material.

Extreme care was taken to ensure that the texture measurements were always conducted in the same area in the middle of the sample gage length for the entire tensile strain range up to $48 \%$. On the other hand, the EBSD mapping of the selected area located in the middle of the gage length was only conducted up to a tensile strain of $32.3 \%$; as the indexing rate at this strain level had dropped significantly to $\sim 55 \%$. Thereafter, the increased surface roughness of the tensile sample precluded further EBSD mapping on the same surface. Consequently, the sample was repolished after $48 \%$ tensile strain and an additional EBSD map was acquired at a considerably improved indexing rate of $\sim 85 \%$.

For the texture measurements, the $\{111\},\{200\}$ and $\{220\}$ incomplete $\left(\alpha=0^{\circ}-85^{\circ}\right)$ pole figures were collected using a PANalytical X'pert-PRO MRD goniometer equipped with a $\mathrm{Cu}$ tube and operating at $40 \mathrm{KV}$ and $\sim 45 \mathrm{~mA}$. The orientation distribution functions (ODFs) were calculated via series expansion using ResMat. While the texture intensities $\left(f_{e}(g)\right)$ along the $\alpha$ and $\langle 100\rangle$-fibers were calculated conventionally along their ideal skeleton lines, the intensities along the $\langle 111\rangle$ fibre were determined in Matlab using a $10^{\circ}$ deviation limit from the ideal orientations. 
EBSD was undertaken on a JEOL-JSM7001F field emission gun (FEG) - scanning electron microscope (SEM) fitted with a Nordlys-II(S) camera and the Oxford Instruments AZtec acquisition software suite and operating at $15 \mathrm{KV}, \sim 2 \mathrm{nA}$ and $15 \mathrm{~mm}$ working distance. A step size of $0.1 \mu \mathrm{m}$ was maintained constant for all the maps. The area of the initial EBSD map at $0 \%$ tensile strain was $80 \times 60$ $\mu \mathrm{m}^{2}$.

In all EBSD maps, subgrain structures are defined by a minimum of 3 pixels and are bounded by misorientations $(\theta) \geq 2^{\circ}$. Consequently, $2^{\circ} \leq \theta<15^{\circ}$ misorientations are defined as low angle grain boundaries (LAGBs) whereas the total high angle grain boundaries (THAGBs) comprise HAGBs $\left(15^{\circ} \leq\right.$ $\theta \leq 57.5^{\circ}$ ) and twin boundaries (TBs). The annealing or deformation twin boundaries are defined as $\Sigma 3$ $=60^{\circ}\langle 111\rangle$. The maximum tolerance of the misorientation angle $(\Delta \theta)$ from the exact axis-angle relationship was identified by the Palumbo-Aust criterion (i.e., $\Delta \theta \leq 15^{\circ} \Sigma^{-5 / 6}$ ) [25] with a tolerance limit of $6^{\circ}$.

The maps were divided into three subsets comprising (sub)grains belonging to the $\langle 111\rangle,\langle 110\rangle$ and $\langle 100\rangle$ fibres with a $15^{\circ}$ deviation from their ideal orientations. A fourth subset, termed as the 'Others', includes grains that do not belong to any of the above three subsets.

For the whole map as well as for the aforementioned four subsets, the intragranular local misorientation between each pixel and its surrounding neighbourhood (in a $3 \times 3$ matrix) was calculated and the mean value was assigned to that pixel. Misorientations over $2^{\circ}$ were excluded in order to negate the misorientations associated with (sub)grain boundaries. The relative frequency of the intragranular local misorientation distribution was fitted to a log-normal (L-N) probability distribution function such that:

$$
f\left(\theta_{L o c}\right)=\frac{1}{\sqrt{2 \pi} w \theta_{L o c}} \exp \frac{\frac{-\left(\ln \theta_{L c} / \theta_{c}\right)^{2}}{2 w^{2}}}{c^{2}}
$$

where, $w$ and $\theta_{c}$ are the width and median of the L-N distribution of $\theta_{L o c}$. The associated mean $\left(\theta_{m}\right)$ and standard deviation $\left(\mu_{\theta}\right)$ are calculated as follows:

$$
\begin{gathered}
\theta_{m}=\theta_{c} \exp \left(\frac{w^{2}}{2}\right) \\
\mu_{\theta}=\theta_{c} \exp \left(\frac{w^{2}}{2}\right) \sqrt{\exp \left(w^{2}-1\right)}
\end{gathered}
$$




\section{Visco-plastic self-consistent modelling}

The macroscopic stress-strain response and the texture evolution during the uniaxial tensile loading of crystalline materials can be simulated using the VPSC model. In this model, each grain is treated as an ellipsoidal visco-plastic inclusion embedded in and interacting with an anisotropic homogeneous effective medium which represents the 'average' environment seen by each grain.

The VPSC model formulation is detailed in previous publications [16-18, 20] and only a brief description of the hardening at the single crystal level is given here as the relevant hardening parameters require assignment. The response of the individual grains is described by activating the various deformation systems $(s)$ at predetermined values of the critical resolved shear stress (CRSS). In each grain, the CRSS evolves with the total accumulated shear strain $\left(\Gamma=\sum_{s} \Delta \gamma^{s}\right)$ by following an extended Voce hardening rule such that [26]:

$$
\tau_{c r}^{s}=\tau_{0}^{s}+\left(\tau_{1}^{s}+\theta_{1}^{s} \Gamma\right)\left[1-\exp \left(-\frac{\theta_{0}^{s} \Gamma}{\tau_{1}^{s}}\right)\right]
$$

where, $\tau_{0}^{s}$ and $\tau_{1}^{s}$ are the initial and back extrapolated critical resolved shear stresses and $\theta_{0}^{s}$ and $\theta_{1}^{s}$ are the initial and final asymptotic hardening rates associated with a deformation system $(s)$. It should be noted that the employed VPSC code does not account for the local intragranular stress concentration which leads to twin nucleation. Consequently, $\tau_{c r}^{s}$ (Eq. 4) for the twinning systems is regarded as a characteristic stress for twin propagation [17, 27].

With respect to latent hardening effects, the activities on the various slip and twinning systems harden each other according to:

$$
\dot{\tau}^{s}=\frac{d \tau_{c r}^{s}}{d \Gamma} \sum_{s^{\prime}} h^{s s^{\prime}} \dot{\gamma}^{s^{\prime}}
$$

where, $h^{s s^{\prime}}$ is the latent hardening matrix which empirically accounts for the obstacles that new dislocations (or twins) associated with the activity of system ( $s$ ) represent for the propagation of dislocations (or twins) on a system $(s)$ [28]. The hardening matrix has diagonal values (or the self hardening) of 1 . The off-diagonal values (or the latent hardening) can be adjusted such that activation of a deformation system (twinning in the present TWIP steel case) on a particular plane contributes to the hardening of the non-coplanar slip or twin systems.

The VPSC simulations that compare the bulk experimental textures with their predicted counterparts use 5000 discrete orientations calculated from the initial experimental texture. In order to account for the elongated morphology associated with the presence of annealing twins in the fully 
recrystallised microstructure, the initial grain shape was described as an ellipsoidal inclusion whose axes along the RD: TD: ND directions had the ratio 3: 1: 1 . The above is based on the grain aspect ratio of 2.9 returned from the initial EBSD map and is calculated by considering twin boundaries as grain boundaries.

Grains were paired at random at the beginning of a simulation and made to co-rotate to improve strain compatibility at grain boundaries by using the grain co-rotation scheme [29]. This scheme allows grains with the same initial orientation but different neighbouring grains to follow different reorientation paths during deformation. Consequently, the overall texture evolution is slowed down during the simulation.

The correspondence between the experimental and the VPSC simulated textures is assessed by the normalised texture index $\left(\hat{T}^{d}\right)$ parameter. The $\hat{T}^{d}$-value of the difference ODF $\left(T^{d}=f_{s}(g)-f_{e}(g)\right)$ quantifies the variation between a simulated texture $\left(f_{s}(g)\right)$ and an experimental texture $\left(f_{e}(g)\right)$ by:

$$
\hat{T}^{d}=\frac{T^{d}}{T}=\frac{\int\left[f_{s}(g)-f_{e}(g)\right]^{2} d g}{\int\left[f_{e}(g)\right]^{2} d g}
$$

where, $T$ is the texture index [30] of the reference experimental texture $\left(f_{e}(g)\right)$. Whereas the $T^{d}$ term indicates the magnitude of difference between any two ODFs, the normalised parameter $\hat{T}^{d}$ has the advantage of allowing direct comparisons between different reference textures. Here smaller $\hat{T}^{d}$ values indicate a more accurate texture prediction. To this end, previous investigations have suggested that $\hat{T}^{d}$ values between $0.1-0.2$ indicate good accuracy of the texture simulation [31, 32].

In order to simulate the changes in the macroscopic response and texture of TWIP steel, three modelling cases have been applied and detailed in the following subsections. It is emphasised that these modelling cases have been specifically constructed to examine individual aspects of TWIP steel deformation behaviour. The fact remains that the physical reality of deformation accommodation is the result of the concurrent, competing and complex interactions between perfect and/or partial slip and twinning.

\subsection{Case I: Twinning as a directional slip mechanism}

Case I treats twinning as a directional slip mechanism. While a twin system requires a CRSS to be activated, it is only operative in one direction (along the Burgers vector of the twin) such that the CRSS must be positive. In all constructs of Case I, twinning is not associated with a reoriented volume and 24 
$\{111\}\langle 110\rangle$ perfect slip systems (counting both forward and reverse slip directions) and 12 $\{111\}\langle 112\rangle$ forward twinning systems are used in the simulations.

Case I consists of three constructs that are denoted as Cases I-A, I-B and I-C and are defined as follows:

- Case I-A is where all the values of the hardening matrix (diagonal and off-diagonal values) are set to 1 such that all deformation systems contribute equally to the hardening of each other.

- Case I-B investigates the twin barrier effect on the slip activity and texture evolution. The latent hardening parameters of the non-coplanar slip systems is assumed to be 5 times harder than coplanar slip systems, i.e., $h^{s s^{\prime}}=1$ for systems $(s)$ and $\left(s^{\prime}\right)$ having the same slip/twin plane and $h^{s s^{\prime}}=5$ for $s$ and $s^{\prime}$ with different planes. In other words, Case I-B imposes strong non-coplanar latent hardening on the slip systems only due to twinning activity. Lebensohn et al. [28] applied a similar construct during the VPSC simulation of rolling texture in low stacking fault energy, fcc brass.

- Case I-C examines the twin barrier effect on both, slip and twinning activity and the associated texture evolution. Here the latent hardening parameters of non-coplanar slip and twin systems is assumed to be 5 times harder than coplanar systems. Thus, Case I-C imposes strong noncoplanar latent hardening on both, slip and twin systems due to twinning activity.

\subsection{Case II: The volume effect of twinning}

Case II utilises the predominant twin reorientation scheme [20] in order to account for the volume effect of twinning on texture evolution. This scheme is a statistical approach wherein twinned grains are fully reoriented to their predominant twin orientation after a certain threshold volume fraction of a grain has undergone twinning. For Case II, $24\{111\}\langle 110\rangle$ perfect slip systems (counting both forward and reverse slip directions) and $12\{111\}\langle 112\rangle$ forward twinning systems were used in the simulations. Here all the values of the hardening matrix (diagonal and off-diagonal values) are set to 1 such that all deformation systems are assumed to contribute equally to the hardening of each other (i.e., no latent hardening is accounted for). Beyerlein et al. [27] also used the predominant twin reorientation scheme to assess the twin volume effect during the VPSC modelling of the rolling texture in Ag-Cu cast eutectic nano-composite.

\subsection{Case III: Partial slip}

Case III assesses the contribution of deformation faulting or slip by partials to texture evolution. Case III is based on the idea originally suggested by Hu et al. [33] to explain the transition from Copper 
to Brass -type textures in cold-rolled low stacking fault energy fcc materials. The mechanism involves glide on fixed $\{111\}$ slip planes in either the conventional $\langle 110\rangle$ or partial $\langle 112\rangle$ directions. Following the same idea, Chin [34] also highlighted the possible contribution of deformation faulting to the wire texture development in low stacking fault energy materials as the widening of the separation between the partials switches the effective slip direction from $\langle 110\rangle$ to $\langle 112\rangle$.

In all constructs of Case III, the grain reorientation caused by the predominant twin reorientation scheme is suppressed. This implies that the partials contribute to deformation accommodation via slip such that the texture evolves without a twinning volume effect [27]. For Case III, $24\{111\}\langle 110\rangle$ perfect slip systems and $24\{111\}\langle 112\rangle$ partial slip systems were used in the simulations. Here all the values of the hardening matrix (diagonal and off-diagonal values) are set to 1 such that all deformation systems are assumed to contribute equally to the hardening of each other.

Case III consists of two constructs that are denoted as Cases III-A and III-B and are defined as follows:

- Case III-A imposes dominant partial slip such that the $\{111\}\langle 110\rangle$ perfect slip systems are given an initial CRSS $\left(\tau_{0}^{s}\right)$ value twice that of the $\{111\}\langle 112\rangle$ partial slip systems. A similar approach was adopted by Beyerlein et al. [27] during the VPSC modelling of the rolling texture in Ag-Cu cast eutectic nano-composite.

- Case III-B assigns equal initial CRSS $\left(\tau_{0}^{s}\right)$ values for both, the $\{111\}\langle 110\rangle$ perfect slip systems and the $\{111\}\langle 112\rangle$ partial slip systems. In an analogous approach, Kocks and Necker [35] and Raabe [36] used the ratio between the CRSS for slip in the net $\langle 112\rangle$ and $\langle 110\rangle$ directions as a floating parameter (adjusted between $2 / \sqrt{3}$ and 0.96) to simulate the Brass -type cold rolling texture.

In summary, while all Case I constructs treat twinning as a directional slip mechanism, Case I-B examines the twin barrier effect on slip and Case I-C examines the twin barrier effect on slip and twinning systems. On the other hand, Cases II and III assess the volume effect of twinning and the contribution of partial slip to texture evolution, respectively.

\section{Results}

\subsection{Mechanical behaviour}

Fig. 1a shows the true stress versus strain curve and the work hardening rate versus the true strain up to the ultimate tensile strength. The red circles in Fig. 1a depict the points where the tensile test was interrupted for EBSD and XRD measurements. In agreement with our previous work on the same 
TWIP alloy [24], an ultimate tensile strength of $1080 \mathrm{MPa}$ and a total elongation of $\sim 66 \%$ were recorded. Compared to [24], the slight differences in the shape of the work hardening curve reported here is due to the differences in the tensile sample geometry. Whereas a flat cross-section and nonASTM standard dimensions were used for the tensile sample of this study, round cross-sections and ASTM standard dimensions were used in [24].

\subsection{Microstructure characterisation}

The band contrast maps track the evolution of the microstructure with progressively greater tensile strain (Fig. 2). The fully recrystallised initial microstructure is characterised by nearly equiaxed grains (when twin boundaries are not considered as grain boundaries) and a high fraction of $\Sigma 3$ annealing twin boundaries. This high fraction of annealing TBs returns an intense peak at $60^{\circ}$ of the misorientation distribution histogram (Fig. 3a) and constitutes $\sim 50 \%$ of the grain boundary area fraction (Fig. 3b). The recrystallised grain size with and without considering twin boundaries as grain boundaries is $\sim 2.6$ and $\sim 5.2 \mu \mathrm{m}$, respectively.

As seen in Figs. 2a, 2b and 3, there is no discernible difference in the microstructure after straining to $0.14 \%$ since it corresponds to a stress of $\sim 210 \mathrm{MPa}$; which is lower than the macro-yielding point at $\sim 290 \mathrm{MPa}$. At 2.3\% (Fig. 2c), striations in the form of single lines or parallel packets evolve preferentially in grains with orientations close to $\langle 111\rangle$ and $\langle 110\rangle$. These striations originate mostly from the grain and/or twin boundaries; while in fewer instances, they were observed to start and finish within a grain. As highlighted by the yellow ovals in Fig. 2c, striations intersecting each other were also found in a few grains.

The density of the striations increases progressively upon tensile straining to $7.2 \%$ and $20.9 \%$. At a tensile strain of $20.9 \%$, some of the striations are indexed as deformation twin boundaries (yellow arrows in Fig. 2e). In localised areas, these newly indexed twin boundaries present either as primary twins (solid yellow arrow) or as intersecting secondary twins (dashed yellow arrow).

The clear white areas in Figs. 2d, e and f are unindexed regions of the EBSD maps as a result of the increasing surface roughness of the sample. This is ascribed to the orange-peel effect induced by the anisotropic nature of plastic strain inside the grains [37]. With increasing tensile strain, grains start popping out of the plane of the tensile sample surface forming peaks and valleys. This indicates that a significant part of the strain is accommodated at the grain boundaries [38]. Consequently, since the EBSD map indexing rate dropped drastically to 55\% after $32.3 \%$ tensile strain (Fig. 2f), further EBSD mapping at $48 \%$ tensile strain was not possible without repolishing and re-mapping the surface at approximately the same position along the gauge length (Fig. 2g). 
The change in the misorientation distribution with tensile strain is shown in Fig. 3a. With increasing strain and in conjunction with the formation of low angle boundaries (Figs. 2b-f), the area fraction of LAGBs continues to increase and is associated with a decrease in HAGBs and $\Sigma 3$ TBs (Fig. 3b). The decline in the population of the twin boundaries with increasing tensile strain (Figs. 3a and b) can be attributed to: (i) dislocations accumulating at the annealing TBs with increasing tensile strain such that the twin/matrix misorientation relationship progressively decays as a result of the increasing local lattice rotations needed to maintain strain compatibility [39]. This point is illustrated by the yellow rectangles in Figs. $2 \mathrm{~d}$ and e such that the annealing TBs up to $7.2 \%$ tensile strain (Figs. 2a-d) are identified as regular HAGBs at a tensile strain of $20.9 \%$ (Fig. 2e). The deviation from the exact $60^{\circ}\langle 111\rangle$ relationship increases with greater tensile strain until it exceeds the employed tolerance limit of $6^{\circ}$ (following the Palumbo-Aust criterion). Hence, such boundaries are no longer identified as TBs but are rather recognised as regular HAGBs. Accordingly, and despite the evolution of deformation twins, the area fraction of TBs generally reduces with increasing tensile strain. (ii) Alternatively, the diminishing population of TBs could also be attributed to the masking effect associated with the increasing frequency of LAGBs (Figs. 3a and b).

It is noted here that the EBSD map after 32.3\% tensile strain returned higher THAGB and lower LAGB fractions compared to the EBSD map of the repolished surface after $48 \%$ tensile strain. The greater error associated with the data at $32.3 \%$ tensile strain is a natural consequence of the poor indexing rate (particularly at grain boundaries) and the increased surface roughness caused by the tensile test. In order to highlight this fact, the data points related to the EBSD map at $32.3 \%$ tensile strain are presented as outliers (with the aid of unfilled markers) in Figs. 3b and d.

The EBSD technique inherently accounts for the variation in the intragranular misorientation such that grains with a higher density of geometrically necessary dislocations record larger intragranular local misorientation values within their boundaries [40, 41]. Here a higher density of geometrically necessary dislocations indicates greater strain heterogeneity within a particular grain (or for a particular crystallographic orientation). The change in the relative frequency distribution of the intragranular local misorientation angle with increasing tensile strain is given in Fig. 3c. With increasing tensile strain, the relative frequency distribution of $\theta_{m}$ shifts towards higher intragranular local misorientation values along with a larger standard deviation ( $\mu_{\theta}$ calculated from Eq. 3 ). The mean intragranular local misorientation values ( $\theta_{m}$ calculated from Eq. 2) of the various fibres are shown in Fig. 3d. From a tensile strain of $20.9 \%$, the change in the $\theta_{m}$-value of the different fibres follows the order $\langle 110\rangle>\langle 111\rangle>\langle 100\rangle$.

Once again the effect of the poor indexing at 32.2\% tensile strain is clearly seen as erroneously higher intragranular local misorientation values are recorded compared to the data from the EBSD 
map of the repolished surface after $48 \%$ tensile strain (Figs. $3 c$ and $\mathrm{d}^{1}$ ). Such errors highlight the limitation of in-situ and ex-situ EBSD deformation studies that track microstructure evolution at the same free surface. Consequently, the boundary and local misorientation data estimated from the EBSD maps of a free surface that is becoming increasingly rougher with greater strain should be interpreted with great care.

\subsection{Texture evolution}

The ideal texture components of fcc materials are shown schematically for the $\phi_{2}=0^{\circ}, 45^{\circ}$ and $65^{\circ}$ ODF sections in Fig. 4 and Table 1. The $\langle 111\rangle,\langle 100\rangle$ and $\langle 110\rangle$ fibres are superimposed on the ODF sections with a spread of $15^{\circ}$ around their ideal skeleton lines. The evolution of the bulk experimental texture (acquired via XRD) with increasing tensile strain is given in Fig. 5. The variation in the intensity $(f(g))$ of the individual texture components along the various fibres is illustrated in Figs. 6a-c.

The fully recrystallised condition is characterised by a weak texture with a maximum intensity of 2.2 and a texture index of 1.17. Typical of fcc materials in general, uniaxial tension resulted in the development of the characteristic $\langle 111\rangle$ and $\langle 100\rangle$ double fibre texture parallel to the loading axis. At $48 \%$ tensile strain (which corresponds to the ultimate tensile strength), the maximum texture intensity lies on the $\langle 111\rangle$ fibre $(f(g)=7.7)$ as compared to the $\langle 100\rangle$ fibre which records a maximum $f(g)=4.6$.

As seen in Fig. 5a, the starting texture comprises an $\alpha$-fibre (extending from the Goss (G) to the Rotated Goss (Rt-G) orientations along $\phi_{1}=0^{\circ}$ to $90^{\circ}$ at $\Phi=45^{\circ}, \phi_{2}=0^{\circ}$ ) as well as the Cube (C), Copper $(\mathrm{Cu})$ and S orientations. All of the above orientations are carried over from the prior cold rolling of the TWP steel to $42 \%$ thickness reduction [42].

With greater tensile strain, a pronounced increase in the intensity of the A orientation is seen in the $\alpha$ and $\langle 111\rangle$-fibre plots (Figs. 6a, b) and is associated with a concurrent decrease in the Rt-G component. The Brass (B) orientation also slightly shifts towards the A orientation with increasing tensile strain such that it is nearly incorporated into the intensity peak of the A orientation at $48 \%$ tensile strain (Fig. 6a). While a significant increase in the texture intensity of all orientations occurs along the $\langle 111\rangle$ fibre (Fig. 6b), the $\mathrm{C}$ and $\mathrm{G}$ orientations along the $\langle 100\rangle$ fibre strengthen to a lesser extent (Fig. 6c).

In order to quantify the stability of the various orientations during tensile loading, the volume fraction of each texture component is normalised against its corresponding initial volume fraction and

\footnotetext{
${ }^{1}$ It is noted that the $\langle 110\rangle$ data point at $48 \%$ tensile strain is absent as the grains have completely rotated towards the $\langle 111\rangle$ and $\langle 100\rangle$ fibres by this point.
} 
plotted as a function of tensile strain in Fig. $6 \mathrm{~d}$. The $\mathrm{Cu}, \sim \mathrm{S}$ and $\mathrm{A}$ orientations belonging to the $\langle 111\rangle$ fibre exhibit the largest increases in volume fraction whereas relatively smaller increases in volume fraction are noted for the $C$ and $G$ orientations of the $\langle 100\rangle$ fibre. The volume fraction of the $B$ orientation was found to remain nearly stable throughout the tensile test such that only a slight decrease in its volume fraction is seen after $48 \%$ strain. This could be ascribed to the proximity between the A and B orientations in Euler space leading to an over estimation of the volume fraction of the B orientation. On the other hand, the volume fraction of the Rt-G orientations lying on the $\langle 110\rangle$ fibre diminishes rather rapidly with increasing tensile strain.

\subsection{VPSC simulations}

The Voce hardening parameters in Eq. 4 were adjusted until good agreement with the macroscopic stress-strain, work hardening and the experimental texture was achieved (Figs. 1b, 1c and 7). The parameters utilised for each modelling case construct are listed in Table 2.

In general, the work hardening is slightly under and over predicted at low $(<\sim 0.04)$ and high ( $>$ $\sim 0.35$ ) strains, respectively (Fig. 1c). This result is qualitatively similar to the work hardening predictions from the multi-site modelling used in Ref. [14]. While good correspondence is obtained in all the modelling cases, it is noted that Case I-C under predicts the work hardening in the intermediate strain range.

Fig. 7 compares the VPSC simulated $\phi_{2}=0^{\circ}, 45^{\circ}$ and $65^{\circ}$ ODF sections for the various constructs of Cases I, II and III at $48 \%$ tensile strain with Fig. 5 g. The texture indices $(T)$ and the normalised difference indices of the DODF $\left(\hat{T}^{d}\right)$ for the different tensile strains are given in Table 3.

Cases I-A and II yield very similar predictions. Both cases quantitatively capture the evolving texture such that the relative intensities of their $\langle 111\rangle$ and $\langle 100\rangle$ fibres are well predicted (Figs. 7a and d) and their corresponding $\hat{T}^{d}$-values are low (Table 3).

Imposing the twin barrier effect on the non-coplanar slip systems in Case I-B returns only qualitative agreement with the experimental texture. The Case I-B simulation has two notable deficiencies: (i) the texture spread between the $\mathrm{G}$ and $\mathrm{A}$ orientations along the $\alpha$-fibre is absent, and (ii) the predicted maximum intensity and texture index values are lower than their experimental counterparts. The latter is due to the slowing down of texture evolution as a direct consequence of hindering perfect slip activity via the imposition of latent hardening. Compared to Case I-B, better correspondence between the simulated and experimental textures is obtained in Case I-C when latent hardening is imposed on the non-coplanar slip and twin systems (Fig. 7c and Table 3). 
In contrast to the experimental observations, the dominant partial slip imposed in Case III-A results in a more intense $\langle 100\rangle$ fibre and a relatively weaker $\langle 111\rangle$ fibre (Fig. 7e). The activation of perfect slip along with partial slip in Case III-B improves the simulation such that the $\langle 111\rangle$ fibre strengthens while the intensity of the $\langle 100\rangle$ fibre decreases.

In Figs. 8a and b, the relative activities of perfect slip (Fig. 8a) and partial slip and twinning (Fig. 8b) are given as a function of tensile strain for the various constructs of Cases I, II and III. Similar activity is returned in Cases I-A and II such that a monotonic decline in twinning is noted for increasing values of tensile strain. Case I-B expectedly returns trends that are the opposite of those seen in Cases I-A and II. Hindering perfect slip via the imposition of latent hardening on the non-coplanar slip systems in Case I-B results in a monotonic increase in twinning activity with greater tensile strain. In Case I-C, the imposition of non-coplanar latent hardening on both slip and twinning systems significantly reduces twinning activity (compared to Case I-B) such that only small and approximately linear increments are noted with greater tensile strain. In Case III-A, partial slip is the dominant deformation mode as the perfect slip activity is almost completely restricted by the latter's double CRSS value (Fig. 8a). Alternatively, in Case III-B, when equal CRSS values are assigned to both perfect and partial slip systems, the perfect slip activity linearly decreases while the partial slip activity linearly increases with greater tensile strain (Figs. 8a and b).

Since Case II is the only modelling case that utilises the predominant twin reorientation scheme, the volume fraction of twins as well as the grains that get fully reoriented due to twinning can be calculated as a function of the tensile strain (Fig. 8c). At $48 \%$ tensile strain, the volume fractions of twins and reoriented grains were $9.1 \%$ and $1.7 \%$, respectively.

\section{Discussion}

\subsection{The observation of in-grain striations}

At $2.3 \%$ tensile strain, striations were observed to evolve preferentially within grains with orientations close to $\langle 111\rangle$ and $\langle 110\rangle$ in the form of single lines or parallel packets. An increase in the tensile strain to $20.9 \%$ resulted in some of these striations being indexed as deformation twin boundaries (indicated by the yellow arrows in Fig. 2e). In this regard, there are two possibilities related to the provenance of the in-grain striations.

Firstly, previous studies $[7,43]$ have pointed out that the thickness of deformation twins at low strain levels is in the order of tens of nanometres such that they cannot be crystallographically detected via EBSD due to spatial resolution limitations. With increasing strain, the twinning activity 
increases such that the twins stack into relatively thick bundles which can eventually be detected by EBSD.

Secondly, transmission electron microscopy (TEM) investigations on low stacking fault energy austenitic steels have frequently reported the formation of stacking faults at the early stages of deformation. Feaugas [44] detected planar dislocation structures comprising pile-ups and stacking faults in a 316L austenitic stainless steel at tensile strains $<1.5 \%$. Stacking faults were also observed in the austenite grains of transformation induced plasticity steel at a tensile strain of $0.5 \%$ [45]. Prior to the observation of deformation twinning, stacking faults in the form of widely separated partial dislocations or as long and narrow straight features have been detected in a Fe-18Mn-0.6C-1.5Al TWIP steel at $4 \%$ tensile strain [46]. During the tensile loading of Fe-30Mn-3Al-3Si TWIP steel, the microstructure was found to be initially dominated by stacking faults and dislocation arrays at $0.19 \%$ strain; with limited deformation twinning observed thereafter at $3 \%$ tensile strain [47].

As opposed to other EBSD studies on TWIP steel tensile deformation behaviour [7, 43], the present work involves tracking the same set of grains during uniaxial tension. This has allowed us to follow the changes in the striations from unindexed individual or packeted parallel lines at $2.3 \%$ tensile strain to indexed twins at 20.9\% tensile strain (Figs. 2c and yellow arrows in Fig. 2e). The above observation agrees more with the mechanism proposed in [48] wherein the divergence of dissociated partial dislocations to an infinite separation distance with increasing tensile strain was presumed to lead to twin formation. Thus, the striations first detected at $2.3 \%$ tensile strain are more attributable to stacking faults forming preferentially at the grain and twin boundaries rather than merely interpreting them as thin, unindexed twins.

In this regard, further TEM -based characterisation that: (i) conclusively evidences the observed striations as stacking faults at low tensile strains, and thereafter (ii) tracks their evolution into twins at higher tensile strains, needs to be undertaken. However, such experiments are beyond the scope of the present study.

\subsection{The dependence of the intragranular local misorientation on grain orientation}

The highest values of intragranular local misorientation are seen in the grains that belong to the $\langle 110\rangle$ fibre (Fig. 3d). It is also noted that at $20.3 \%$ tensile strain, the unindexed regions of the EBSD map mostly comprised deformed grains with orientations aligned along the $\langle 110\rangle$ fibre. The high intragranular local misorientation in grains along the $\langle 110\rangle$ fibre was previously reported in austenitic stainless steel [49]. This phenomenon was attributed to grain-to-grain interaction such that the presence of stable $\langle 111\rangle$ oriented grains results in preferential plastic flow in the unstable $\langle 110\rangle$ 
oriented grains [50]. An example of grain-to-grain interaction is given in Section 5.5 in the second set of VPSC simulations.

Compared to all other orientations, the $\langle 100\rangle$ fibre records the lowest intragranular local misorientation values (Fig. 3d). Near $\langle 100\rangle$ oriented grains with relatively lower local misorientation effects were also inferred from TEM work on Al subjected to uniaxial tension [51, 52]. In those studies, planar geometrically necessary dislocations were absent from the $\langle 100\rangle$ oriented grains such that they only contained dislocation cells. Following their observations of dislocation cells in the $\langle 100\rangle$ oriented grains of a Fe-22Mn-0.6C TWIP steel, Gutierrez-Urrutia et al. [53] postulated that the dependence of the dislocation cell formation on the crystallographic orientation was similar in both low and high stacking fault energy materials. In this regard, the exact mechanism for dislocation cell formation in low and high stacking fault materials has not yet been fully elucidated and requires further investigation [53].

It is noted that previous studies have tended to correlate the dependence of the intragranular local misorientation on grain orientation to the Taylor factor; such that grains with larger Taylor factor values possess a higher density of geometrically necessary dislocations $[40,50]$. However, such correlations ignore: (i) the inability of the Taylor model to account for deformation inhomogeneities and, (ii) that the Taylor factor is only a measure of a material's current state without considering its prior deformation history [54]. Both these factors are important considerations when evaluating the development of geometrically necessary dislocations.

\subsection{The VPSC model predictions}

Whereas the adjustment of the Voce hardening parameters of Eq. 4 allowed for satisfactory predictions of the stress-strain response in all modelling cases, the work hardening curves and the predicted texture are more sensitive to the relative activity of the various deformation modes.

With respect to texture evolution, the best agreement with the experimental ODFs was obtained for the directional slip approach of Case I-A and the predominant twin reorientation scheme of Case II. Both cases predict the development of a stronger $\langle 111\rangle$ fibre through relatively high $\{111\}\langle 110\rangle$ perfect slip activity (Figs. 7a, 7d and 8a). Thus, both cases emphasise the limited effect of twinning on bulk texture evolution during uniaxial tension up to $48 \%$ tensile strain. This is further verified by the small Case-II -predicted values for the volume fractions of twins (9.1\%) and reoriented grains (1.7\%) at $48 \%$ tensile strain (Fig. $8 \mathrm{c}$ ).

Here our estimate of the twin volume fraction from Case II is also in general agreement with literature based estimates of the limited twin volume fraction in TWIP steels. Barbier et al. [7] 
estimated a $9 \%$ twin volume fraction in a Fe-22Mn-0.6C TWIP steel after 55\% tensile strain. A twin volume fraction of $\sim 9 \%$ was also measured using combined SEM and TEM -based observations in a Fe-Mn TWIP alloy after 50\% tensile strain [55].

When the latent hardening of slip and twin systems are considered as proposed in Case I-C, sufficient perfect slip systems are activated such that satisfactory texture predictions are obtained. Since the values for the normalised difference indices of the DODF in Case I-C $\left(\hat{T}^{d}=0.15\right)$ are just slightly higher than Cases I-A $\left(\hat{T}^{d}=0.12\right)$ and II $\left(\hat{T}^{d}=0.13\right)$, the possible contribution of latent hardening to the overall texture evolution cannot be negated.

As opposed to Hu et al. [33], Leffers and Ray [56] negated the partial slip mechanism (or Case III of this study) as a separate Copper to Brass texture transition theory in their recent review. Their objections are based on the possible slip patterns of the partials such that: (i) if slip by one partial is followed by the corresponding partial on the same slip plane, the result would be equivalent to perfect slip along the $\langle 110\rangle$ direction. In this argument, the consideration of partial slip would not alter the texture evolution as it would be dictated solely by perfect slip. (ii) Alternatively, if slip by one partial is followed by the slip of the same partial on a neighbouring slip plane, this would lead to deformation twinning such that a significant part of the matrix would be reoriented by twinning. This scenario is, in effect, similar to Case II of the present study. (iii) The random operation of partials would result in an anomalously high density of stacking faults. In agreement with the objection in point (iii), it was also pointed out in $[33,35]$ that the operation of single $\langle 112\rangle$ partials would result in excessive faulting and limited strain accommodation. Consequently, Kocks and Necker [35] suggested that net $\langle 112\rangle$ slip is possible by the simultaneous glide of two $\langle 110\rangle$ perfect slip vectors.

To this end, the VPSC simulations using the partial slip approach of Case III-A (which considers the CRSS for perfect slip to be twice the value of CRSS for the partial systems) are unsatisfactory as they return an intense $\langle 100\rangle$ fibre (Fig. 7e). On the other hand, in Case III-B (which considers the CRSS for perfect slip to be equal to the value of CRSS for the partial systems) the texture predictions were significantly improved. However, Cases I-A, II and I-C (in order) still provide better overall agreements with the bulk texture. The above result indicates that during uniaxial tensile loading, the CRSS in the $\langle 112\rangle$ direction has to be higher than the CRSS in the $\langle 110\rangle$ direction in order to enforce dominant perfect slip. This conclusion is in contrast with an earlier modelling study on austenite wherein a slightly lower CRSS value in the $\langle 112\rangle$ direction $\left(\mathrm{CRSS}_{<112>}=0.96 \times \mathrm{CRSS}_{<110>}\right)$ was used to reproduce a Brass -type cold rolling texture [36].

In summary, the evolution of crystallographic texture during the uniaxial tension of TWIP steel is well captured by the VPSC model. The limited correspondence between the experimental and 
simulated results of earlier investigations is more likely to be associated with the improper description of the hardening parameters of the various deformation systems.

\subsection{Orientation stability during uniaxial tension}

In this sub-section, three additional sets of VPSC simulations were undertaken in order to understand: (i) how individual texture components evolve with increasing tensile strain, and (ii) how their interaction with each other affects the development of the overall bulk texture. All simulations were performed using a fictitious discretised ODF of 1000 grains such that a $5^{\circ}$ deviation was imposed around each ideal fcc orientation.

Case II hardening parameters have been utilised in all of the following simulations (Table 2). The choice of Case II was based on the facts that: (i) its bulk texture predictions were equivalent to Case I$\mathrm{A}$, and (ii) the ODFs from either Cases I-A and II are in general agreement with the experimental data as they both enforce dominant perfect slip. It is also emphasised that we are only interested in the development of the texture and not in the macroscopic stress-strain response. Therefore, the exact values of the single crystal hardening parameters are not relevant [29].

It is understood that that the exact rotation path of individual grains depends on their interaction with the local surrounding homogeneous effective medium [16-18, 20]. Thus, for the first set of VPSC simulations on individual orientations, the interaction effect with differently oriented neighbouring grains is unaccounted for. Nonetheless, and in an average sense, the model predictions provide a qualitative means to track the rotation (or stability) of individual orientations with increasing tensile strain.

Since all the orientations along the $\langle 111\rangle$ and $\langle 100\rangle$ fibres were found to remain stable during uniaxial tension up to $48 \%$ strain, they have been omitted from the figures. On the other hand, the Rt-G orientation on the $\langle 110\rangle$ fibre undergoes significant reorientation during uniaxial tension. As seen in Fig. 9a, the Rt-G orientation rotates from the $\langle 110\rangle$ pole (solid blue circles at $0 \%$ tensile strain) towards the $\langle 111\rangle$ pole (solid red circles at $48 \%$ tensile strain). The reorientation effect is very well captured along the $\alpha$-fibre; as a shift in texture intensity from the Rt-G orientation towards the A component is clearly noted in Fig. 9b².

In the second set of VPSC simulations, the interaction effects of neighbouring grains were quantified by coupling together various ideal orientations from different fibres and subjecting them to

\footnotetext{
${ }^{2}$ Although the ultimate tensile strength was reached at $48 \%$ tensile strain during experimental tensile testing, the VPSC simulations in Fig. 9b were extended up to $70 \%$ tensile strain in order to confirm the trend of reorientation from the Rt-G orientation towards the A orientation.
} 
uniaxial tension up to $48 \%$ strain. The coupled orientations included: (i) G ( $\langle 100\rangle$ fibre) and A ( $\langle 111\rangle$ fibre), (ii) G ( $\langle 100\rangle)$ and Rt-G ( $\langle 110\rangle)$, and (iii) A ( $\langle 111\rangle)$ and Rt-G ( $\langle 110\rangle)$.

As seen in Fig. 9c, the coupling together of the G-A orientations did not result in any rotation of the individual orientations at $48 \%$ tensile strain. When the G-Rt-G orientations are coupled together, the Rt-G orientation rotates toward the A orientation in behaviour that is reminiscent of the individual orientation simulations of Fig. 9b. Thus the reorientation of the Rt-G component by the presence of the $G$ orientation can be negated. Alternatively, the coupling together of the A-Rt-G orientations accelerates the rotation of the Rt-G component towards the A orientation such that the Rt-G is completely reoriented at the A position by $48 \%$ tensile strain. This strong grain-to-grain interaction between the orientations belonging to the $\langle 111\rangle$ and $\langle 110\rangle$ fibres corroborates the argument in Section 5.3 and [50]. Therein, the relatively higher intragranular local misorientations in the unstable $\langle 110\rangle$ grains were ascribed to them undergoing preferential plastic flow as a result of their interactions with the stable $\langle 111\rangle$ orientations.

A third VPSC simulation was performed using the EBSD data from the 'Others' subset (at $0 \%$ tensile strain) as the initial texture. The strongest orientation in the initial ODF of the 'Others' subset is located at (132)[221] (at $\phi_{1}=25^{\circ}, \Phi=50^{\circ}, \phi_{2}=20^{\circ}$ ); which belongs to the $\langle 221\rangle$ fibre parallel to the tensile axis. The $\langle 221\rangle$ fibre is nearly half-way between the $\langle 111\rangle$ and $\langle 110\rangle$ poles on the inverse pole figure (IPF). The predicted texture after $48 \%$ tensile strain is shown in Fig. $9 \mathrm{~d}$ using $\phi_{2}=0^{\circ}, 15^{\circ}$ and $45^{\circ} \mathrm{ODF}$ sections. The most intense orientation is predicted for (143)[32 2$]$ (at $\phi_{1}=35^{\circ}, \Phi=52^{\circ}, \phi_{2}=$ $\left.15^{\circ}\right)$; which is close to the $\langle 111\rangle$ fibre $^{3}$.

It is worthwhile to compare the three sets of VPSC predictions with the in-situ 3D-XRD experiment of Poulsen et al. [57] and Winther et al. [58] on $\mathrm{Al}$ subjected to 6\% tensile strain. Therein, the rotation behaviour of 95 grains was tracked and correlated with their initial orientations within the bulk polycrystal. In those studies, the IPF was divided into four regions exhibiting different rotation behaviours such that: (i) grains at the $\langle 110\rangle$ corner rotate towards the $\langle 111\rangle-\langle 100\rangle$ line. This is in agreement with our first set of VPSC predictions discussing the rotation of the Rt-G orientation towards the A orientation with greater tensile strain. (ii) Grains along the $\langle 111\rangle-\langle 100\rangle$ line rotate towards the $\langle 111\rangle$ corner. This line connects the orientations between $\mathrm{G}$ and A (through $\mathrm{B}$ ) along the $\alpha$-fibre. Here it is noted that the B orientation does not belong to any of the three fibres. However, the VPSC simulation of the individual B orientation to $48 \%$ tensile strain indicated a slight rotation towards the A component (i.e., towards the $\langle 111\rangle$ fibre). This prediction is in accordance with the

\footnotetext{
${ }^{3}$ When the VPSC simulation was theoretically extended to $70 \%$ tensile strain, the orientations that belong to the 'Others' subset rotate even further towards the $\langle 111\rangle$ pole.
} 
experimental observations stipulated in point (ii). (iii) Grains in between $\langle 111\rangle-\langle 110\rangle$ poles rotate towards the $\langle 111\rangle$ corner. Here the grains of the 'Others' subset initially lie in-between the $\langle 111\rangle-\langle 110\rangle$ poles and mostly belong to the $\langle 221\rangle$ fibre. With increasing tensile strain, the third VPSC simulation predicts that the orientations of the 'Others' subset rotate towards the $\langle 111\rangle$ fibre (Fig. 9d). (iv) Grains at the $\langle 100\rangle$ corner exhibit the smallest rotation amongst all other orientations and have no preferred rotation direction. This corroborates the predicted stability of the $\mathrm{G}$ and $\mathrm{C}$ orientations that belong to the $\langle 100\rangle$ fibre (also Fig. 9c).

In summary, the response of the various orientations subjected to uniaxial tension can be generally classified as follows: (i) stable orientations along the $\langle 100\rangle$ fibre which do not strongly interact with or affect other orientations, (ii) stable orientations along the $\langle 111\rangle$ fibre which strongly interact with other unstable orientations, and (iii) unstable orientations along the $\langle 110\rangle$ fibre whose reorientation is accelerated as a result of their interactions with the orientations along the $\langle 111\rangle$ fibre.

It is emphasised that the above comparison between TWIP steel and $\mathrm{Al}[57,58]$ is strictly valid for uniaxial tension only. It follows that varying the deformation path, for example compression or rolling, would obviously lead to changes in twinning activity and its slip-confining effect.

Moreover, the qualitative correspondence between our VPSC -based predictions of the rotation behaviour in TWIP steel and the experimental work of Poulsen et al. [57] and Winther et al. [58] on Al does not imply that the work hardening and/or texture formation mechanisms in low and high stacking fault energy materials are the same. Inherent differences in the work hardening and/or texture formation in low and high stacking fault energy materials do exist and are ascribed to the variation in the slip character, the occurrence of twinning and the associated latent hardening effects. In this regard, the achievement of simultaneous quality predictions of both, strain hardening and texture in low and high stacking fault energy materials would require different unique sets of Voce parameters. For more quantitative comparisons between low and high stacking fault energy materials, detailed experiments similar to that in Refs. $[57,58]$ are required over extended strain ranges in order to account for the strain effect as well. The latter experiments are however, beyond the scope of the present work.

\section{Conclusions}

Using a combination of EBSD, XRD and VPSC modelling, the microstructure and texture evolution of a fully recrystallised TWIP steel undergoing interrupted uniaxial tensile testing has been characterised. The results show that: 
(1) The present TWIP steel records the development of the double fibre texture which is characteristic of fcc materials. A relatively stronger $\langle 111\rangle$ and a weaker $\langle 100\rangle$ fibre were noted parallel to the tensile axis.

(2) Grains with orientations along the $\langle 100\rangle$ fibre return the lowest values of intragranular local misorientation indicating a low density of geometrically necessary dislocations. Alternatively, grains with unstable orientations along the $\langle 110\rangle$ fibre exhibit the highest values of intragranular local misorientation as a result of their interaction with the stable $\langle 111\rangle$ oriented grains.

(3) The VPSC model has been successfully used to simulate the macroscopic stress-strain response and texture evolution. The simulations verify the dominant role of perfect slip and the limited volume effect of twinning on the texture formation. A possible role for latent hardening on the non-coplanar slip and twinning systems cannot be negated.

(4) The stability of various orientations subjected to uniaxial tension was examined via the VPSC model. The simulations predict that orientations along the stable $\langle 100\rangle$ fibre do not affect unstable orientations whereas the orientations along the stable $\langle 111\rangle$ fibre strongly affect the unstable orientations along the $\langle 110\rangle$ fibre.

\section{Acknowledgments}

This work was conducted with part funding from the 2011 UOW Research Centre (URC) Small Grants scheme and the Engineering Materials Institute (EMI). The authors acknowledge the use of the JEOL-JSM7001F FEG-SEM at the UOW Electron Microscopy Centre purchased with financial support from the Australian Research Council (LE0882613). The authors are grateful to Prof. D. B. Santos of the Federal University of Minas Gerais, Brazil for providing the source material. The authors also thank Dr. Carlos Tomé of Los Alamos National Laboratory, USA for the VPSC program and for his valuable advice in developing the modelling constructs of this paper.

\section{References}

[1] Grassel O, Kruger L, Frommeyer G, Meyer LW. Int. J. Plast. 2000;16:1391.

[2] English AT, Chin GY. Acta Metall. 1965;13:1013.

[3] Barrett CS, Massalski TB. Structure of metals: Oxford ; New York : Pergamon, 1980.

[4] Stout MG, Kallend JS, Kocks UF, Przystupa MA, Rollett AD. In: Kallend JS, Gottstein G, editors. Proceedings of the 8th International Conference on Textures of Materials (ICOTOM 8). Santa Fe: TMS, Warrendale, 1988. p.479.

[5] Mecking H. Textures in metals. In: Wenk H-R, editor. Preferred orientation in deformed metals and rocks: an introduction to modern texture analysis. Orlando: Academic Press, 1985. p.267. 
[6] Yang P, Xie Q, Meng L, Ding H, Tang Z. Scr. Mater. 2006;55:629.

[7] Barbier D, Gey N, Allain S, Bozzolo N, Humbert M. Mat. Sci. Eng. A 2009;500:196.

[8] Gutierrez-Urrutia I, Zaefferer S, Raabe D. Mat. Sci. Eng. A 2010;527:3552.

[9] Jiménez JA, Frommeyer G. Mater. Charact. 2010;61:221.

[10] Allain S, Chateau JP, Bouaziz O. Mat. Sci. Eng. A 2004;387-389 143.

[11] Bouaziz O, Allain S, Scott C. Scr. Mater. 2008;58:484.

[12] Bouaziz O, Guelton N. Mat. Sci. Eng. A 2001;319-321:246.

[13] Barbier D, Favier V, Bolle B. Mat. Sci. Eng. A 2012;540:212.

[14] Dancette S, Delannay L, Renard K, Melchior MA, Jacques PJ. Acta Mater. 2012;60:2135.

[15] Van Houtte P, Li S, Seefeldt M, Delannay L. Int. J. Plast. 2005;21:589.

[16] Lebensohn RA, Tomé CN. Acta Metall. Mat. 1993;41:2611.

[17] Lebensohn RA, Tomé CN. Materials Science and Engineering: A 1994;175:71.

[18] Tomé CN, Lebensohn RA. Self consistent homogeneization methods for texture and anisotropy. In: Raabe D, Roters F, Barlat F, Chen L-Q, editors. Continuum Scale Simulation of Engineering Materials. Berlin: Wiley-VCH, 2004. p.473.

[19] Prakash A, Hochrainer T, Reisacher E, Riedel H. Steel Res. Int. 2008;79:645.

[20] Tomé CN, Lebensohn RA, Kocks UF. Acta Metall. Mat. 1991;39:2667.

[21] Kalidindi SR. J. Mech. Phys. Solids 1998;46:267.

[22] Yan K, Carr DG, Callaghan MD, Liss K-D, Li H. Scr. Mater. 2010;62:246.

[23] De Cooman BC, Kim J, Lee S. Scr. Mater. 2012;66:986.

[24] Santos DB, Saleh AA, Gazder AA, Carman A, Duarte DM, Ribeiro ÉAS, Gonzalez BM, Pereloma EV. Mat. Sci. Eng. A 2011;528:3545.

[25] Palumbo G, Aust KT. Acta Metall. Mat. 1990;38:2343.

[26] Tomé CN, Canova GR, Kocks UF, Christodoulou N, Jonas JJ. Acta Metall. 1984;32:1637.

[27] Beyerlein IJ, Mara NA, Bhattacharyya D, Alexander DJ, Necker CT. Int. J. Plast. 2011;27:121.

[28] Lebensohn RA, Tomé CN, CastaÑeda PP. Phil. Mag. 2007;87:4287.

[29] Tomé C, Necker C, Lebensohn R. Metall. Mater. Trans. A 2002;33:2635.

[30] Bunge HJ. Texture Analysis in Materials Science: Mathematical Methods. London: Butterworth, 1982.

[31] Engler 0, Crumbach M, Li S. Acta Mater. 2005;53:2241.

[32] Engler O. J. Appl. Cryst. 2009;42:1147.

[33] Hu H, Cline RS, Goodman SR. In: Margolin H, editor. American Society of Metals Seminar on Recrystallization, Grain Growth and Textures. Metals Park, Ohio, 1966. p.295.

[34] Chin GY. In: Grewen J, Wassermann G, editors. Textures in research and practice. Clausthal: Berlin: Springer-Verlag, 1969. p.51.

[35] Kocks UF, Necker CT. In: Andersen SI, Bilde-Sorensen JB, Lorentzen T, Pedersen OB, Sorensen NJ, editors. 15th Risø International Symposium on Materials Science 'Numerical Prediction of Deformation Processes and the Behaviour of Real Materials'. Risø National Laboratory, Roskilde, Denmark, 1994. p.45.

[36] Raabe D. Acta Mater. 1997;45 1137.

[37] Reed-Hill RE, Abbaschian R. Physical metallurgy principles. Boston: PWS-Kent, 1992.

[38] Vercammen S. Ph.D. Thesis. Processing and tensile behaviour of TWIP steels - Microstructural and textural analysis, vol. Ph.D. Thesis: KU Leuven, Belgium, 2004.

[39] Mishra S, Tiwari S, Kumar A, Hector L. Metall. Mater. Trans. A 2012;43:1598.

[40] Brewer LN, Field DP, Merriman CC. Mapping and Assessing Plastic Deformation Using EBSD. In: Schwartz AJ, Kumar M, Adams BL, Field DP, editors. Electron Backscatter Diffraction in Materials Science. Springer, 2009. p.251.

[41] Gazder AA, Sanchez-Araiza M, Jonas JJ, Pereloma EV. Acta Mater. 2011;59:4847.

[42] Saleh AA, Pereloma EV, Gazder AA. Mat. Sci. Eng. A 2011;528:4537.

[43] Jin J, Lee Y. Mat. Sci. Eng. A 2009;527:157.

[44] Feaugas X. Acta Mater. 1999;47:3617

[45] Rafaja D, Krbetschek C, Borisova D, Schreiber G, Klemm V. Thin Solid Films 2012; In press.

[46] Beladi H, Timokhina IB, Estrin Y, Kim J, De Cooman BC, Kim SK. Acta Mater. 2011;59:7787.

[47] Zhang X, Sawaguchi T, Ogawa K, Yin F, Zhao X. 8th European Symposium on Martensitic

Transformations, Article Number: 05029. Prague, Czech Republic: EDP Sciences, 2009.

[48] Byun TS. Acta Mater. 2003;51:3063. 
[49] Ojima M, Adachi Y, Suzuki S, Tomota Y. Acta Mater. 2011;59:4177.

[50] Merriman CC, Field DP, Trivedi P. Mat. Sci. Eng. A 2008;494:28.

[51] Huang X, Hansen N. Scr. Mater. 1997;37:1.

[52] Winther G. Mat. Sci. Eng. A 2008;483-484:40.

[53] Gutierrez-Urrutia I, Raabe D. Acta Mater. 2011;59:6449.

[54] Gazder AA, Saleh AA, Pereloma EV. Scr. Mater. 2011;65:560.

[55] Soulami A, Choi KS, Shen YF, Liu WN, Sun X, Khaleel MA. Mat. Sci. Eng. A 2011;528:1402.

[56] Leffers T, Ray RK. Prog. Mater. Sci. 2009;54 351.

[57] Poulsen HF, Margulies L, Schmidt S, Winther G. Acta Mater. 2003;51:3821.

[58] Winther G, Margulies L, Schmidt S, Poulsen HF. Acta Mater. 2004;52:2863. 